\title{
La religion communicationnelle
}

Communicative Religion

La religión comunicacional

\section{Hubert Knoblauch}

\section{CpenEdition}

\section{Journals}

Édition électronique

URL : http://journals.openedition.org/assr/26142

DOI : $10.4000 /$ assr.26142

ISSN : $1777-5825$

Éditeur

Éditions de l'EHESS

Édition imprimée

Date de publication : 20 octobre 2014

Pagination : 83-104

ISBN : 978-2-7132-2433-1

ISSN : 0335-5985

\section{Référence électronique}

Hubert Knoblauch, «La religion communicationnelle», Archives de sciences sociales des religions [En ligne], 167 | juillet-septembre 2014, mis en ligne le 20 octobre 2017, consulté le 19 avril 2019. URL : http://journals.openedition.org/assr/26142 ; DOI : 10.4000/assr.26142 


\section{Hubert Knoblauch}

\section{La religion communicationnelle ${ }^{1}$}

Alors que le paradigme de la sécularisation a longtemps dominé les débats sur le devenir de la religion dans les sociétés occidentales, particulièrement en Europe, nous assistons depuis peu à des formes de résurgence, que l'on nomme tantôt " retour du religieux ", tantôt « re-sacralisation ", tantôt même, de manière plus polémique, "dé-sécularisation ». Ce changement a pris une dimension mondiale depuis la fin de la Guerre froide et va de pair avec le déclin des idéologies séculières telles que le socialisme, le nationalisme et plus récemment le capitalisme libéral. L'Europe est souvent qualifiée d'exception en raison de sa forte sécularisation (G. Davie, 2002; P. Berger, 1999). Néanmoins, nous avons de solides raisons de considérer que les sociétés européennes sont aussi le théâtre d'une certaine revitalisation de la religion (H. Knoblauch, 2008), même si celleci ne suit pas le même modèle qu'ailleurs. En effet, contrairement à ce que l'on observe en Afrique et en Amérique du Nord comme du Sud, les nouveaux mouvements chrétiens ont en Europe un succès plus limité. En ce qui concerne l'islam, dont le dynamisme est attesté, il demeure principalement une religion de migrants et de leurs descendants (d'une importance certes croissante en raison de la transformation des sociétés européennes en sociétés d'immigration). Dans ce contexte européen, les dynamiques de revitalisation relèvent aussi largement du développement d'une nouvelle forme de religiosité que l'on qualifie souvent de spiritualité.

Deux enquêtes récentes appuient cette affirmation ${ }^{2}$. La première (E. Barker, 2004), menée dans huit pays européens auprès de 11000 participants montre que si $37 \%$ des sondés se considèrent comme à la fois "spirituels et religieux ", $12 \%$ s'affirment "spirituels mais pas religieux » (ce chiffre varie entre 2 et $24 \%$ selon les pays), $15 \%$ « religieux mais pas spirituels » et $35 \%$ « ni spirituels, ni religieux ». La seconde enquête (S. Huber, 2009) inclut 19 pays (y compris postcommunistes et non européens) et interroge 21000 répondants sur leurs expériences « théistes » ou "panthéistes ». En dépit des limites méthodologiques inhérentes à l'usage de telles catégories, l'un des résultats significatifs pour notre

1. Traduit de l'anglais par Linda Hennig et Matthieu Amat. Je remercie Mathias Blanc pour ses commentaires précieux et Anne-Sophie Lamine pour l'adaptation du texte au lecteur français.

2. Pour une analyse secondaire détaillée de ces données voir H. Knoblauch (2010, 2012). 
propos est qu'un très grand nombre de personnes ne se considérant pas comme religieuses rapportent néanmoins des expériences panthéistes, c'est le cas pour plus de la moitié d'entre elles en Allemagne, en Suisse, au Brésil, en Inde ou en Indonésie. Des formes de religiosité non théistes et non institutionnelles sont donc attestées aussi bien au sein de groupes religieux qu'en dehors d'eux. On ne considèrera donc pas ici la spiritualité comme opposée à la religion mais plutôt comme transgressant les frontières du religieux, et c'est en cela notamment qu'on montrera qu'elle peut être qualifiée de "populaire ».

En regard des anciennes organisations religieuses, qui gardent néanmoins une importance sociale et économique significative, les nouvelles formes de religiosité peuvent donc difficilement être définies en termes d'institutions religieuses spécifiques, comme le fait la sociologie classique de la religion. C'est à propos de ces transformations que le sociologue allemand Thomas Luckmann (1967) emploie sa fameuse expression de la "religion invisible ». Il insiste ainsi sur le fait que la religion devient de plus en plus une affaire privée, qui relève de décisions individuelles. Cette religion «privatisée » est exposée aux mécanismes du marché, au syncrétisme individuel et au "bricolage ".

Cette thèse de la privatisation est contestée par José Casanova (1994), qui considère que l'on observe au contraire une dé-privatisation de la religion. Le sociologue hispano-américain analyse les processus par lesquels la religion sort de la sphère privée, prétend à sa légitimation dans la sphère publique et s'invite dans ses débats en défendant des droits, des libertés ou encore des formes traditionnelles de vie, contre les attaques bureaucratiques (J. Casanova, 1994: 65 sq.). La religion devient alors un acteur dans la sphère publique, et l'auteur affirme que nous allons vers des "religions publiques ${ }^{3}$ ", qui peuvent se situer à divers niveaux de l'ordre social et dont les effets se repèrent depuis la révolution islamique et la montée du fondamentalisme protestant américain dans les années 1970, jusqu'à la montée globale des mouvements religieux et l'attention nouvelle qu'ils suscitent à partir du tournant $\mathrm{du} \mathrm{XXI}^{\mathrm{e}}$ siècle, en passant par le rôle joué par l'Église catholique dans l'effondrement du régime communiste polonais.

En plus d'être pertinente pour la description de nouveaux phénomènes religieux, la notion de «spiritualité populaire » permet de dépasser la contradiction apparente entre les thèses de Luckmann (privatisation) et de Casanova (de-privatisation). Casanova a certes raison de dire que la religion est un acteur institutionnel de plus en plus important dans la société civile, mais il faut néanmoins préciser que le retour du religieux ne se réduit pas à la religion institutionnalisée, qui est, elle, toujours sur le déclin. Luckmann a certes raison d'affirmer que les formes syncrétiques et individualisées du religieux se propagent indépendamment des congrégations et des Églises, il faut néanmoins ajouter qu'aujourd'hui

3. Celles-ci peuvent se situer à trois niveaux de l'ordre social (ibid., p. 218-219) : niveau étatique (Églises d'État), niveau de la société politique (partis démocrates-chrétiens, mobilisation de l'Église catholique polonaise sous le régime communiste) ; niveau de la société civile (militants anti-avortement, lettres pastorales des évêques sur l'euthanasie). 
les religions sont tout sauf publiquement invisibles. Les sujets religieux privés deviennent des objets publics ${ }^{4}$ et tout ce qui est public est susceptible d'une appropriation individuelle ${ }^{5}$. La notion de "religion publique » proposée par Casanova ne recouvrant que la religion organisée, je lui préfère celle plus large de spiritualité ou religion «populaire»(H. Knoblauch, 2012).

La notion de "populaire» rejoint certes celle de la Théorie critique, des cultural studies ou de la sociologie de la culture, mais elle y ajoute des dimensions que nous expliciterons en détail. En bref, cette notion permet d'abord de prendre en considération le fait que les limites entre le public et le privé sont transgressées par les transformations sociales de la communication (y compris la communication religieuse). Elle inclut aussi la transformation fondamentale de la religion dans une société qui se distingue de plus en plus de la société industrialisée, fonctionnellement différenciée et "moderne » que nous avons l'habitude de considérer comme « sécularisée ».

Pour saisir cette transformation de la religion, nous avons besoin d'une théorie de la religion prenant en compte à la fois la religion institutionnalisée et ses formes privatisées. S'esquisse alors la possibilité d'une théorie faisant converger entre elles la notion phénoménologique de transcendance, la sociologie schützienne de la connaissance et une approche plus récente, le "constructivisme communicationnel » (R. Keller, H. Knoblauch, J. Reichertz, 2012). Pour ce faire, on discutera d'abord la théorie de la religion de Thomas Luckmann. La notion de transcendance - permettant d'aborder aussi bien la religion institutionnelle que les spiritualités qui lui sont plus ou moins extérieures - peut être construite en combinant les approches phénoménologique, anthropologique et sociologique. On appréhendera ensuite la dimension sociologique de la transcendance. La notion d'intersubjectivité développée par Alfred Schütz permet d'élargir l'agir communicationnel habermassien et de montrer comment la transcendance est structurée par la communication. Enfin on présentera les traits principaux de la spiritualité populaire contemporaine, dans ou hors du religieux, ainsi qu'à ses frontières. Quelques perspectives de recherche ouvertes résulteront de cette nouvelle approche.

\section{Approches de la transcendance}

La théorie de la «religion invisible » de Thomas Luckmann s'appuie sur plusieurs traditions en sociologie des religions. Si Durkheim, avec sa distinction entre le sacré et le profane, demeure une référence majeure, c'est la théorie weberienne de l'action qui constitue le fondement principal de la théorie de la

4. Que l'on pense aux débats sur le halal, sur le ramadan ou sur les signes religieux, mais aussi du côté non institutionnel aux diverses polémiques sur les thérapies alternatives, par exemple.

5. Comme l'expérience du seuil de la mort qui était un «tabou » et qui est devenue un genre populaire dans les mass media et sur internet (H. Knoblauch, 2011), ou encore le culte des anges, qui s'appuie sur une production culturelle de masse. 
construction sociale de la réalité (P. Berger et T. Luckmann, 1966). L'action ${ }^{6}$ est définie par le sens subjectif que lui attribue l'acteur; elle devient sociale lorsque ce sens est orienté vers autrui. Dans ses études sur la relation entre les religions mondiales et les éthiques économiques, Weber montre que les mises en forme de sens religieux peuvent influer l'action économique de manière décisive pour le changement social.

Aussi impressionnante que soit la manière dont Weber (1988 [1920]) a élaboré sa thèse, à travers la relation entre le protestantisme et le capitalisme, Schütz critique le manque d'une définition claire de la notion centrale de sens et propose de recourir à la phénoménologie de Husserl pour développer une analyse qui constituera l'arrière-fond de la définition de la transcendance chez Luckmann.

Il nous faut aussi tenir compte d'une autre influence qui marque la théorie de Berger et Luckmann, celle de l'anthropologie philosophique. Cette dernière s'appuie sur la comparaison d'études menées dans diverses disciplines anthropologiques, pour tenter d'identifier les traits généraux de l'espèce humaine, la conditio humana, et sa différence par rapport à d'autres formes de vie. Cette influence est notamment repérable dans la définition de la religion que propose Luckmann dans la première version de La Religion invisible ${ }^{7}$, en la référant à la transcendance. Selon lui, la transcendance ne désigne pas un domaine séparé, opposé à l'immanence des actions terrestres, mais ce qui transforme des organismes vivants en êtres humains capables d'attribuer du sens et de se distancier des processus de perception, des instincts et d'un apprentissage de type strictement « behavioriste ». La transcendance relie donc à autre chose, non "factuellement » soumis à la perception. C'est sur la base de cet être-en-relation [relatedness] que l'être humain peut s'éprouver lui-même, éprouver [experience ${ }^{8}$ ] son corps en tant que corps.

Avant d'élaborer l'aspect phénoménologique de la notion de transcendance, il faut préciser qu'elle n'est pas synonyme de religion. Même si Luckmann souligne que ces deux notions renvoient au même contexte de sens, la religion stricto sensu est définie comme un "point de vue sur le monde » (Weltansicht) constituant un " cosmion ", un ordre universel indépendant des expériences vécues de l'individu' 9 .

6. N.d.t. : ou «activité » si l'on se réfère à la traduction des travaux de Max Weber aux éditions Plon.

7. Le livre a été publié dans une version allemande dès 1963 avant d'être réécrit en anglais en 1966.

8. La notion de transcendance est liée à ce que Plessner (1975) considéra à partir des années 1920 comme le trait le plus spécifique de la condition humaine, la "positionnalité excentrique » [exzentrische Positionalität] vers le corps propre, ce qui donne lieu à la distinction entre le " corps» [Körper $]$ et la " chair » [Leib].

9. Selon P. Berger et T. Luckmann (1966: 128-129) la religion est un des « univers symboliques " qui produisent de la légitimation dans divers champs institutionnels en les rendant "subjectivement signifiants " et en leur accordant une "validité cognitive ". 
Luckmann établit un lien entre les notions anthropologique et phénoménologique de transcendance. L'organisme humain, anthropologiquement défini, est capable de se mettre en relation avec quelque chose qui n'est pas donné sur le moment par l'expérience. Cette relation est qualifiée d' "apprésentation » par Husserl (1972 [1939]). Cela signifie, en bref, que la conscience associe au fait empirique quelque chose qui n'est pas donné. C'est dans la mesure où l'apprésentation offre l'expérience de quelque chose de non présent, qu'il y a transcendance. La transcendance ne renvoie donc pas - en général - à un autre monde ni ne signifie le contraire de "l'immanence ", dans une opposition qui, comme chez Luhmann (2000), par exemple, se substitue à la distinction entre sacré et profane. En étant rattachée à l'expérience, la transcendance fait partie de l'intentionnalité de l'apprésentation.

Les deux notions de transcendance (anthropologique et phénoménologique) semblent compatibles. L'idée phénoménologique de transcendance permet en outre de distinguer plusieurs niveaux de transcendance (A. Schütz, 1962 ; A. Schütz et T. Luckmann, 1989 et T. Luckmann, 1991). D’abord, les «petites transcendances » relient à ce qui est absent dans le temps et dans l'espace (ce qui est de l'autre côté de la cloison, ce que je ferai demain à mon bureau). Ensuite, les " transcendances intermédiaires » renvoient aux aspects inaccessibles des autres sujets (ce que pense ou éprouve mon interlocuteur ou toute autre personne). Enfin, les "grandes transcendances » sont des expériences qui renvoient à des réalités non accessibles dans le monde de notre vie quotidienne, celui dans lequel nous pouvons agir sur notre environnement et communiquer avec les autres (le rêve, l'extase mystique, etc.). La religion est alors, selon Schütz (1962) une " province [spécifique] de sens ${ }^{10}$ », le sens étant intégré à la fois dans la structure de la conscience et dans celle des symboles. Alors que Berger (1974) se tourne vers cette approche tendanciellement substantialiste de la religion, Luckmann (1991), lui, se focalise (de manière fonctionnaliste) sur ce qui compte socialement comme religieux et en tirera la distinction de trois niveaux de transcendance, que nous discuterons plus loin.

En plus des dimensions anthropologique et phénoménologique de la transcendance, on trouve dans la théorie de Luckmann les indices d'une troisième dimension, proprement sociologique. Dans cette dernière perspective, la transcendance ne repose ni sur l'organisme humain, ni sur un accomplissement de la conscience, mais elle constitue un trait essentiel des interactions sociales : la distance par rapport à sa propre expérience est un résultat de la distance vis-à-vis de l'expérience de l'autre dans l'interaction. Il s'agit bien là de la dimension sociologique de la transcendance.

10. Pour A. Schutz, le social est composé de réalités multiples auxquelles correspondent des provinces de sens (notion qu'il emprunte à William James), parmi lesquelles on peut citer le monde religieux, mais aussi les mondes de la vie quotidienne, des rêves, du jeu enfantin, de la contemplation scientifique ou encore de la folie. 
Alors que la différence entre les notions anthropologique et phénoménologique est seulement une question de degré, la notion sociologique diffère radicalement des deux premières, dans la mesure où elle affirme que l'expérience subjective de la transcendance résulte du processus de construction sociale de la réalité. Le sens des actions, initialement constitué par les acteurs, est aussi construit socialement de telle sorte qu'il devient non seulement indépendant des acteurs mais aussi qu'il les façonne, dans la mesure où il fournit l'assise nécessaire à la définition sociale des identités personnelles. La théorie du constructivisme social constitue de ce fait un défi pour le fondement même de la théorie des transcendances. Si le sens est remplacé par un savoir socialement construit, son analyse se réduira à celle de certaines formes spécifiques de connaissance et les notions de transcendance définies à partir de l'anthropologie ou de la phénoménologie ne seront plus que des « a priori sociohistoriques » (T. Luckmann, 1973), ces formes spécifiques de sens socialement construites dans certaines cultures.

\section{La construction communicationnelle de la transcendance}

Articuler la socialité des transcendances avec la dimension corporelle offre la pertinence d'une approche en terme de constructivisme communicationnel. On se référera pour cela à une idée avancée par Schütz (1966) lorsqu'il critique la primauté de la phénoménologie de la conscience subjective sur la socialité. Alors que Husserl place la constitution «transcendantale » de l'autre dans la conscience subjective, Schütz propose de considérer l'autre comme empiriquement donné ou même comme précédant l'ego (au sens "génétique »). L'analyse du monde social ne doit donc pas commencer avec le sujet mais avec l'intersubjectivité.

[Celle-ci] est la catégorie ontologique fondamentale de l'existence humaine dans le monde et par conséquent de toute anthropologie philosophique. Tant que l'être humain est né d'une mère, l'intersubjectivité et la « relation-sur-le-mode-du-Nous » [we-relationship] demeurent le fondement de toutes les catégories de l'existence humaine. La possibilité de la réflexion sur le soi, la découverte de l'ego, la capacité d'accomplir une epoché quelle qu'elle soit et la possibilité de la communication et du monde communicationnel qui l'entoure, reposent sur l'expérience primordiale de la « relation-sur-le-mode-du-Nous » (A. Schütz, 1966: 82).

Le sens subjectif est fondamentalement social, c'est-à-dire dérivé des autres en tant que "savoir " (il l'est nécessairement par le biais de la communication). Luckmann souligne également le rôle de l'intersubjectivité dans la transcendance :

La mise à distance du flux de sa propre expérience résulte en effet de la participation aux expériences d'un semblable. Ceci est possible seulement par un face à face dans lequel les processus subjectifs de l'un des partenaires se manifestent par des événements dans un temps et un espace communs et deviennent ainsi observables pour l'autre (T. Luckmann, 1991: 83).

En tant que phénomène intersubjectif, la transcendance inclut les autres, leurs corps et, par conséquent, la communication. Elle dépend de la possibilité 
d'éprouver l'alter ego par le corps, d'une manière sensible. Finalement, on peut dire qu'empiriquement, l'action sociale - ce processus fondamental de la société pour Weber, Schütz ou Luckmann - est une action communicationnelle.

Cette inférence constitue un axiome central de ce constructivisme. Cette approche s'appuie sur l'agir communicationnel d'Habermas (1981), mais en diffère sur deux points. Dans la mesure où l'observabilité corporelle empirique de l'action communicationnelle requiert la production de porteurs matériels du sens, (a) on ne peut séparer la " rationalité téléologique " (reliée à la " nature ") de la "rationalité communicationnelle» (reliée aux autres). L'insistance sur la distinction entre nature et société relève plutôt d'un accomplissement culturel spécifique qui s'est généralisé. Cela implique aussi que l' " autre » n’est pas nécessairement un être humain, comme l'écrit Luckmann (1970) en soulignant qu'une action communicationnelle peut être accomplie avec des animaux, des plantes, voire même des pierres, qui s'avèrent des partenaires de communication efficaces au sein de certaines traditions religieuses. Ce n'est donc pas nécessairement l' « autre " qui transcende l'ego, comme l'affirme Csordas (2004) en se référant à Levinas (1979). La diversité des pratiques religieuses montre bien que la transcendance n'est pas forcément personnelle.

Cette considération nous amène au second point de désaccord avec la notion d'agir communicationnel (b). En effet, contrairement à Habermas qui considère le sens comme lié au langage et la revendication de la validité comme ressortant des actes de langage, nous insistons sur le fait que le sens et la transcendance sont aussi impliqués dans la communication corporelle et non linguistique. Tomasello (2008) montre de manière remarquable le rôle du corps dans la constitution du sens et la transcendance, en analysant la communication corporelle dans divers groupes. Alors que les bébés humains et les chimpanzés communiquent de façon coordonnée pour poursuivre leurs buts individuels, les humains de plus de neuf mois révèlent aussi une capacité plus élaborée de communication, que l'on peut illustrer par le pointage du doigt. Les animaux et les bébés échouent à identifier le doigt montré en tant que montrant autre chose. En revanche, les humains réussissent à percevoir ce doigt pointé comme un objet d'attention commun. Comme le dit Tomasello - en utilisant une catégorie de Searle - ils «partagent l'intentionnalité ». Partager l'intentionnalité implique une gamme d'activités, analysées par Schütz (1962a), qui relèvent de l' «intersubjectivité ». Ainsi l'identification du doigt pointé implique que l'action de montrer soit perçue comme une activité par quelqu'un d'autre. Cela présuppose aussi ce que Schütz appelle la réciprocité des perspectives : afin de savoir vers où l'autre pointe le doigt, je dois pouvoir m'imaginer dans la position spatiale de l'autre. En outre, pour concevoir que l'autre est en train de montrer quelque chose à quelqu'un d'autre, je dois «prendre le rôle de l'autre ", comme l'écrit Mead (1964), pour que le «motif-en-vue-de» de la personne opérant cette désignation se transforme en un «motif-parce-que» : je regarde parce que cette personne montre quelque 
chose. Par ailleurs, "comprendre » et accomplir ce pointage impliquent un processus que Cooley (1902) appelle l'effet «miroir » : notre perception visuelle produit une image de miroir inversé ; bien que je ne puisse pas voir mon visage regardant vers l'endroit que quelqu'un montre, je peux voir que l'autre peut me voir regarder.

La réciprocité n'est donc pas ici un postulat normatif (comme dans la théorie de l'agir communicationnel de Habermas) mais un processus de base dans la constitution de l'intersubjectivité. Ce n'est pas seulement une idéalisation effectuée par la conscience. Ainsi que le montre notre exemple, ce processus nécessite un troisième élément qui est indiqué par le rôle du doigt : il est ici question du corps et de ce qui est relié au corps, à savoir des éléments matériels et des objets. Si le rôle du corps et des "objectivations " a déjà été souligné (A. Schütz, 2003 : 268 ; P. Berger et T. Luckmann, $1966: 49$ ), il faut aussi insister sur le fait que l'acte de communication illustré par le pointage n'est pas une forme de sens [meaning] par «signification » [signification] (comme l'est le langage parlé). Il s'agit d'un processus de «travail social » [social working] (A. Schütz 1962a, $\mathbb{4} 4$ ), dans lequel quelque chose au sein de l'environnement communicationnel (ce qui est perçu comme "étant perçu » et donc commun), une objectivation matérielle d'un certain type est reliée - en tant que "causée ", " effectuée » ou " signifiée »à un acte de communication. Cela implique que l'autre et moi-même soyons conscients que chacun perçoit (ou peut percevoir) l'objectivation.

Sans être en mesure d'élaborer la catégorie difficile de l'objectivation et sa relation aux signes (et significations) conventionnels, ceci nous amène à souligner la structure triadique de l'action communicationnelle qui la distingue des " approches dialogiques ${ }^{11}$ » : les « objets » ne sont pas opposés au « sujet »; le sujet, l'autre et les objectivations sont plutôt des catégories relationnelles.

La réciprocité des perspectives étant donnée, la relation triadique illustrée par le pointage du doigt permet de saisir un type de « transcendance ${ }^{12}$ ». La « deixis » du doigt pointé est une apprésentation de quelque chose qui n'est pas « ici » mais « là ». Il s'agit d'une « petite » transcendance, spatiale. Ce type d'apprésentation ne peut être réduit à un processus de la conscience, comme le fait Husserl (1972). Il dépend du sujet et, simultanément, du sujet anticipant l'action des autres. Ces autres, réciproquement, jouent leur rôle, anticipent le point de vue de l'autre et reconnaissent la dimension motivationnelle du fait de montrer, ou, dit autrement, la relation triadique dans son futur proche (transcendance temporelle) lorsque le destinataire se tournera vers ce qui est montré. L'action de montrer ne repose

11. La relation triadique a été mise en avant par K. Bühler (1934) et reprise et adaptée par J. Habermas (1981).

12. Comme l'a montré A. Leroi-Gourhan, la « libération » de la main par la position verticale peut être considérée comme une condition de la signification ; le montrer du doigt, dans son apparente trivialité, peut ainsi avoir une signification évolutionniste. 
donc pas uniquement sur l' "origo » du sujet, elle est relationnelle (W. Hank, 1990). Lorsque le doigt désigne quelque chose qui n'est pas le doigt, la transcendance du sens dépend de la transcendance de la signification [meaningfulness] des performances corporelles.

On commence à percevoir que l'action communicationnelle croise divers types de transcendances (distinguées par A. Schütz et par T. Luckmann), mentionnées dans la première partie. Tandis que la « petite » transcendance du temps et de l'espace constitue la condition préalable de la réciprocité des perspectives, l'orientation vers autrui, comme quelqu'un qui attribue un sens au doigt pointé, correspond à la transcendance "intermédiaire ». Ces transcendances ne sont pas données, elles dépendent des questions suivantes : qui est considéré comme l'autre ? Qu'est-ce qui est considéré comme objectivation dans l'environnement commun?

On peut à présent se demander comment ces formes de transcendance sont liées à la religion. Luckmann $\left(1990^{13}\right)$ considère que la religion peut également reposer sur des transcendances "petites" ou "intermédiaires ". Cela revient à dire que ce qui est considéré comme religieux inclut des activités que Weber qualifierait d'intra-mondaines, comme le fait de chanter, de participer à des rassemblements ou à des œuvres caritatives. Dans ce sens Luckmann nous offre une lecture phénoménologique de ce que d'autres auteurs qualifient de "sécularisation interne » de la religion. L'objectif étant ici d'élaborer une approche sociologique de ce qui est spécifiquement religieux ou, plus exactement, spirituel, on suivra Schütz et Luckmann (1989) lorsqu'ils avancent l'idée que la religion repose sur de "grandes " transcendances. Il faut alors garder à l'esprit que : (a) les transcendances ne sont pas seulement un processus de la conscience, (b) elles ne correspondent pas à des domaines substantiels de la conscience mais plutôt à des constructions historiques spécifiques.

Concernant le premier aspect (a); alors que la phénoménologie considère la transcendance en tant qu'activité accomplie par la " conscience ", ce qui précède suggère de définir aussi la "grande » transcendance par rapport à l'action communicationnelle. Comme on l'a vu, cette dernière implique que nous maîtrisions les «petites » transcendances (du temps et de l'espace) ainsi que les transcendances « intermédiaires » (d'autrui), qui, en raison de la réciprocité, ne peuvent être séparées l'une de l'autre. Alors que ces transcendances font partie de la performance de l'action communicationnelle dans sa structure triadique, l'orientation vers cette structure en tant que totalité peut être considérée comme une " grande » transcendance. En effet, de même que le principe de la religion durkheimienne repose sur une collectivité « trans-individuelle », c'est son unité la plus élémentaire, la relation triadique, qui émerge de l'action communicationnelle.

13. Voir dans ce dossier p. 31-46. 
L'expression sociale de cette unité transcendante est la forme qui repose sur des actions coordonnées réciproquement (mais pas nécessairement «coopérativement »). Forme qui produit des collectivités, voire des États, mais également qui constitue des types de situation et d'action telles que des paroles, des conversations et des événements. Les diverses formes communicationnelles se caractérisent par leur distinction vis-à-vis des autres formes - et en conséquence par leurs relations avec elles. Non seulement elles relient à «quelque chose »(de différent en fonction des formes : un discours religieux n'est pas un discours politique), mais elles sont elles-mêmes orientées par la manière dont les acteurs en usent et les interprètent. Elles sont les moyens par lesquels l'ordre est construit d'une manière qui transcende l'action communicationnelle et simultanément oriente vers elle les acteurs agissants. La société étant construite par des actions communicationnelles, les formes communicationnelles peuvent être perçues comme productions réflexives d'ordre social. Elles sont une manière de produire certaines institutions (religieuses, politiques, juridiques, etc.) et, parallèlement, elles indiquent la spécificité de ces institutions aux acteurs, que ceux-ci soient inclus ou exclus. C'est donc bien au moyen des formes communicationnelles que l'ordre social « en acte » est établi et qu'il est observable, un ordre qui transcende même la situation dans laquelle il est produit.

À propos du second aspect précédemment mentionné, celui des constructions historiques spécifiques (b) ; Durkheim, Schütz et Berger se donnent une définition substantielle de la religion, selon laquelle celle-ci dépend d'une certaine " province de sens " de la conscience qui peut être définie comme un certain style d'expérience ou un "style cognitif " [cognitive style]. La notion de transcendance suggérée ici ne s'écarte pas seulement de la religion en tant que phénomène défini d'abord par la conscience. En affirmant que la " grande " transcendance constitue un point de départ pour la construction communicationnelle de la religion, elle remet également en question l'hypothèse selon laquelle la religion peut être définie de façon substantielle. La religion est évidemment un phénomène sociohistorique. Selon Eisenstadt et Jaspers (1999), la religion, au sens historique étroit, a vu le jour dans «la période axiale » qui court de 800 av. J.-C. à 200 apr. J.-C. Les discussions sur les raisons de sa remarquable dissémination ne sont pas closes, mais ces raisons tiennent probablement à l'un de ses traits spécifiques : la tension croissante entre «transcendance » et « immanence » (en d'autres mots entre le sacré et le profane), qui a soulevé des projets d'actions collectives utopiques. Aussi impressionnante qu'ait été l'expansion de ces « religions historiques ", on peut douter qu'elles offrent un modèle pour les visions du monde des autres cultures. L'échec de la "phénoménologie de la religion » a montré que la comparaison des différents aspects de la religion à travers la culture humaine ne permet de découvrir aucun élément commun. Par conséquent, Asad (2003) a raison de déclarer que la catégorie même de "religion » est une construction occidentale particulière qui s'avère difficile à appliquer à d'autres 
religions mondiales. Bien que Durkheim soit visé par cette critique lorsqu'il projette des catégories occidentales de la religion sur ses "formes élémentaires » (S. Lukes, 1973), son idée de définir la religion par la distinction binaire entre le sacré et le profane s'avère utile pour comprendre les religions provenant de la période axiale. On peut alors constater que la distinction entre les sphères de la transcendance et de l'immanence, qui implique la réification idéologique des grandes transcendances, s'est accompagnée d'une spécialisation sociale des rôles religieux et des savants parallèlement à une spécialisation de la communication et du savoir religieux.

Une des raisons d'adopter comme fondement de la religion (et plus largement de la spiritualité), la notion englobante de transcendance structurée par la communication, est le fait que les dynamiques religieuses ne peuvent pas toutes être expliquées à partir de ce code binaire (sacré/profane ou transcendance/ immanence). Les grandes transcendances ne sont pas forcément structurées par l'opposition du sacré et du profane (et en conséquence par des formes de communication mettant l'accent sur des traits opposés et des distinctions nettes entre les institutions correspondantes). Il est plus adéquat de définir les formes de communication religieuse comme des cadres qui s'entrecroisent, se chevauchent, s'intègrent, se recadrent, se recouvrent ou décrochent. Se focaliser sur l'étude empirique des formes communicationnelles permet alors de poser exactement la question de savoir en quels lieux, comment et par qui les frontières sont contestées, négociées ou tracées, constituant ainsi des « communautés communicationnelles ».

Les religions historiques tendent à constituer des communautés communicationnelles distinctes qui tracent leurs frontières en soulignant explicitement leurs formes spécifiques de communication, par exemple par l'ornementation, la glorification, l'exagération, voir même par des tentatives de nier les formes de communications "communes", comme dans le quiétisme religieux (R. Bauman, 1983). D'après Samarin (1987) la communication religieuse passe par des moyens linguistiques, parfois uniquement par des sons (A. Wilke, O. Moebius, 2011), ou bien par une diversité de genres communicationnels spécialisés, tels que les prières, les sermons, les récitations ou les formules magiques (L. Honko, 1968). Si l'on considère, par exemple, les formes de l'architecture, du langage ou des rituels religieux, on peut constater que les formes de la communication religieuse ne s'expriment pas forcément en opposition à d'autres formes. Ainsi Luckmann a avancé que le sermon moralisateur provient d'un genre communicationnel religieux, transposé dans un cadre " religieux civil ", comme aux États-Unis, ou, dans un cadre plus sécularisé, comme en Allemagne. En ce qui concerne les rituels religieux, M. Bloch (1924), par exemple, a montré comment le charisme religieux de guérison a été longtemps attribué aux rois de France. L'un des traits les plus intéressants des formes communicationnelles est probablement qu'en plus de mettre en lumière l'ordre social qu'elles contribuent à établir, elles en indiquent également les changements. 


\section{Spiritualité populaire ou religion communicationnelle}

Bien que l'on puisse douter du fait que la religion dans les sociétés occidentales médiévales ait été aussi institutionnalisée et spécifique que l'ont soutenu les récits modernes de la sécularisation, de Comte à Parsons (G. Duby, 1978 ; R. Muchembled, 1978), la thèse de l'augmentation de la vitalité et de l'importance sociale de la religion (au sens large, incluant les spiritualités), avancée dans notre introduction, entre vivement en contradiction avec des auteurs qui justifient empiriquement la thèse de l'avancée de la sécularisation (S. Bruce, 2002 ; D. Pollack, 2003). Mais, comme on tente de le montrer, l'idée de spiritualité populaire permet de dépasser cette contradiction tout comme celle de la privatisation.

Dans la mesure où l'on considère ici le terme de religion dans une acception large, il est préférable de lui substituer le terme de spiritualité. La notion de spiritualité s'appuie sur la transcendance, et le qualificatif populaire se réfère à une forme de la communication, telle que nous l'avons précédemment entrevue. Cette notion de spiritualité permet donc de dépasser l'opposition entre le sacré et le profane, ou le séculier et le religieux. En conséquence, la spiritualité populaire est une forme de religion plus séculière dans la mesure où elle ne suit pas uniquement ou même majoritairement le modèle de religion institutionnalisée qui semblait prédominer depuis son établissement en tant qu'organisation rationnelle, qui ne compte pas uniquement sur ses membres mais les compte également. D'un autre côté, la société est devenue plus religieuse dans la mesure où les formes de communication religieuse sont devenues accessibles, sans limitation ou même sans médiation des institutions religieuses, à un nombre croissant de personnes. L'approche en termes d'action communicationnelle facilite l'observation empirique de cette transformation vers la spiritualité populaire, tout comme des transformations récentes qui affectent les religions historiques.

Un aspect important de la popularité est la transgression des limites de la connaissance institutionnalisée. Et c'est bien ce que l'on observe, avec la fin du monopole des mass media (de leurs débuts avec l'invention de l'imprimerie jusqu'aux "Églises électroniques », en passant par la radio et la télévision) sur les moyens de communication. Les grandes organisations religieuses spécialisées ont elles aussi perdu leur hégémonie (que celle-ci s'organise en terme de compétition économique entre institutions religieuses, de champs de légitimité ou de monopoles) sur leurs moyens de communication. En conséquence, le savoir religieux est devenu largement, voire universellement, accessible au public. Ce processus a été préfiguré par la "révolution éducative ", d'abord au sein de la société américaine dans les années 1950 et par la construction sociale d'une «classe savante " depuis les années 1960, elle-même entraînée par les transformations sociotechniques, et liée au déclin rapide de la société industrielle, à l'affaiblissement de la classe ouvrière et à l'importance croissante des femmes dans tous les domaines de la société. La révolution de la communication a produit une expansion de la communication globale avec des conséquences importantes pour la 
religion : le savoir religieux est devenu accessible à l'échelle mondiale, ce qui conduit non seulement à des formes "syncrétiques » de religion (comme en témoignent les nouveaux mouvements religieux fondés après le milieu $\mathrm{du} \mathrm{XX}^{\mathrm{e}}$ siècle et la diffusion du New Age dans la culture populaire), mais aboutit également à la pénétration de formes religieuses variées au niveau mondial dans d'autres sphères de l'action communicationnelle (comme la publicité ou l'art). La "révolution communicationnelle » s'accompagne de changements sociaux considérables, en même temps qu'a lieu ce que je désigne comme la transformation de la religion en spiritualité populaire. L'émergence de celle-ci est un aspect (mais pas le seul) de ce changement, qui affecte et qui est affecté par la transformation des formes de communication.

$\mathrm{Si}$, pour caractériser cette forme de religion, nous employons la notion commune de «populaire » [popular] utilisée habituellement dans le contexte général de la culture, nous pouvons éviter ses ambiguités en distinguant trois formes majeures de popularisation : a) selon P. Burke (1988), la culture populaire désigne diverses formes traditionnelles de culture, parfois rassemblées dans la notion de culture «folklorique ». Les formes modernes de spiritualité «populaire » pourraient être considérées comme l'effet d'une transformation progressive de celle-ci. b) Par ailleurs, le terme culture populaire peut caractériser une "culture de masse ", en référence notamment à la communication par les mass media, à la production industrielle de masse, à la rationalisation des organisations et de l'économie. De ce point de vue, le style sulpicien du « kitsch » catholique ou « l'Église électronique " protestante, qui se sert de la radio et de la télévision, constituent des exemples de forme moderne de spiritualité populaire (H. Knoblauch, 2009 : 79, 215). Cependant, en parlant de spiritualité «populaire » je me réfère (c) à une forme de popularité qui n'est plus déterminée par des formes de communication de masse. Elle correspond, avec l'ajout d'un tréma, à l'allemand «populär» (H. Knoblauch, 2000). D'une part la spiritualité «populaire » utilise de nouvelles structures "médiatisées » de l'action communicationnelle (A. Hepp, 2012), comme les médias sociaux, d'autre part, elle cesse d'être définie en opposition à la culture et à la religion de la classe dominante au sein des sociétés nationales. Cette spiritualité populaire se diffuse dans toutes les classes mais aussi au-delà des sociétés nationales et des aires culturelles. La spiritualité populaire mise en évidence par les " expériences panthéistes » évoquée en introduction constitue ici un exemple de phénomène diffusé dans dix-neuf sociétés nationales, sans distinction ni de classe ni de genre, partout dans le monde et au sein de religions différentes ${ }^{14}$. Contrairement à ce qu'en pensent certains auteurs des cultural studies (J. Fiske, 1989), la spiritualité " populaire» n’est plus essentiellement déterminée, marquée ou influencée par des organisations

14. En France, par exemple, plus de $54 \%$ de la population a connu au moins une expérience "panthéiste" (H. Knoblauch, 2012). 
religieuses ; la communication religieuse dépasse les frontières entre le religieux et le non-religieux d'une manière qui ne permet plus de considérer le sacré et le profane comme une démarcation décisive pour ce qu'on peut appeler la spiritualité.

En un sens, la spiritualité populaire correspond à l'idée de Bourdieu (1985) sur la " dissolution du champ religieux » par de nouvelles formes d'organisation, de services et de professions. D'ailleurs, la notion de populaire ne désigne pas uniquement la transgression des frontières du champ religieux, mais aussi ses résultats et effets qui peuvent être décrits en termes de communication. Bien que l'on doive admettre que la forme de cette transgression ne puisse pas encore être définie, la notion permet déjà d'identifier certaines transformations :

(a) Des formes qui, jusqu'à présent, n'étaient connues que dans la culture populaire deviennent aujourd'hui partie intégrante des organisations religieuses : des objets matériels « relevants » d'un marketing de masse, l'usage d'une musique populaire couvrant toute une gamme de styles et de sujets plus ou moins religieux, ou des formes de médias typiques des mass media - ce sont là quelques exemples de la "popularisation » de la religion.

(b) On observe, dans la nouvelle culture populaire un usage croissant de formes empruntées à la communication religieuse, parfois par de claires références (ainsi la chanteuse Madonna qui utilise la croix dans ses shows ou l'emploi des symboles religieux dans le milieu " gothique »), mais souvent sans distinction claire, comme dans la glorification populaire de l'amour comme transcendance ultime ou encore dans le recours à l'architecture religieuse dans des centres commerciaux ou dans des lieux de vente de voitures. Aussi diverse, riche et omniprésente soit la transgression des formes religieuses de la communication, celleci était déjà préfigurée dans la culture populaire moderne.

(c) De nouvelles formes propres à la spiritualité populaire apparaissent, telles que l' "événementialisation » et la "festivalisation ». Comme l'a montré l'analyse des Journées mondiales de la jeunesse (Forschungskonsortium, 2007), les événements religieux sont organisés de manière rationnelle, avec des méthodes empruntées à la gestion commerciale des événements et dans l'intention explicite de produire des " expériences » qui se distinguent nettement de la routine quotidienne (R. Hitzler, 2011). Les événements, rituels inclus, visent explicitement des états émotionnels et expérientiels du public. Un autre phénomène de transformation des rituels religieux est leur «médiatisation ». Ce terme se réfère - par contraste avec les représentations médiatiques de la religion véhiculées par les mass media - à l'intrusion des médias électroniques dans la vie quotidienne et à leurs effets sur la structure des actions, des interactions et des rituels. Ainsi, l'analyse des messes du pape, notamment lors de ses déplacements, démontre comment les formes de dévotion et de piété religieuse s'organisent par et autour des nouvelles technologies de l'image et de la vidéo, remplaçant des formes plus anciennes (R. Herbrik, H. Knoblauch, 2013). 
L' «événementialisation » et la médiatisation affectent non seulement les formes d'action communicationnelle mais aussi le rôle de l'acteur. En raison des effets "individualisants" de la communication de soi par les mass media (M. Castells, 2009), augmente l'importance des individus en tant que nœuds des structures communicationnelles. Toutefois cette "individualité » est secondaire par rapport à la "subjectivité ». Même dans le cas d'événements communicationnels qui visent à ce que l'individu ait une expérience de la collectivité, c'est avant tout l'authenticité de l'expérience de l'individu qui est visée. L'accent mis sur la subjectivité et l'expérience subjective s'exprime par excellence dans l'immense succès que connaît la spiritualité : il n'est pas nécessaire que les expériences spirituelles prennent des formes hautement individualisées mais elles doivent être émotionnellement et corporellement "authentiques»(H. Knoblauch, 2012). Soulignant la dimension personnelle de la religion, la spiritualité a même envahi le langage courant. Sociologiquement, la spiritualité peut être conceptualisée en référence à ce que Troeltsch (1992 [1912]) considère comme la forme " mystique » de la religion. Le rejet des doctrines et rituels prescrits par la religion organisée est articulé avec la valorisation des expériences de la transcendance, qui peuvent être des rencontres avec des êtres religieux (comme dans les mouvements charismatiques ou évangéliques chrétiens), des pouvoirs impersonnels, des énergies ou encore des dimensions cachées du soi (H. Knoblauch, 2009).

(d) Comme l'exemple de la spiritualité l'illustre, la popularité s'exprime par la diffusion de la spiritualité au travers de la structure sociale des sociétés, des différents niveaux de culture et des différentes religions ${ }^{15}$. Cette diffusion large a une particularité qui doit être mise en avant dans la discussion sur la religion : la distinction entre " religieux " et "spirituel » ne dépend pas de l'affiliation à la religion organisée. L'expérience impersonnelle de " grandes » transcendances peut être trouvée chez de nombreuses personnes " croyantes ", et des expériences de dévotion peuvent être revendiquées par des personnes se considérant comme « non-croyantes ». Par conséquent, la distinction entre des personnes « croyantes » et «non-croyantes ", si importante dans l'analyse sociologique de la « sécularisation ", est dépassée. La spiritualité est donc présente chez nombre de ceux qui se considèrent comme "non-croyants", alors que des formes de ce qui était autrefois qualifié de New Age alternatif ou de spiritualité ésotérique sont visibles au cœur même des organisations religieuses formelles, y compris chez des prêtres (W. Gebhardt, M. Engelbrecht, C. Bochinger, 2005).

Luckmann avait déjà souligné l'importance de la subjectivité dans la « religion invisible ». Il est néanmoins très significatif du processus de popularisation que des sujets « intimes » tels que la mort, la guérison ou le bien-être spirituel (auparavant quasiment monopolisés par la religion chrétienne et les institutions confessionnelles) soient devenus visibles dans la sphère publique. Le nouveau rapport à la mort, les nouveaux rituels qui lui sont liés et l'accompagnement en

15. Voir H. Knoblauch, 2010 et 2012. 
fin de vie se sont ainsi développés presque exclusivement en dehors du champ religieux. Le thème de la "guérison " [healing] illustre bien la transgression non seulement du champ religieux mais aussi du champ médical (H. Knoblauch, 2009).

Alors que la culture populaire a été analysée par opposition à une forme hégémonique représentée par des dispositifs organisationnels, que les formes modernes de la religion sont dominées par des organisations religieuses, celles-ci ont toutefois perdu leur monopole de communication, même sur ces questions qui sont encore marquées du sceau du religieux dans la "mémoire religieuse » (D. Hervieu-Léger, 1993). Cette observation appuie la thèse de la transformation vers une spiritualité populaire et sa dépendance vis-à-vis de la communication et de ses transformations : les institutions religieuses perdent de plus en plus leur monopole sur les moyens de communication religieuse. Cette perte de contrôle communicationnel ne concerne pas seulement les organisations monopolistiques mais toutes les organisations religieuses, qu'elles soient de type Église ou secte. Le savoir religieux (y compris les actions orientées selon un sens religieux et les rituels) est désormais accessible à chacun d'une manière qui échappe de plus en plus au contrôle des organisations religieuses, des experts religieux et de l'ordre établi du champ religieux.

Enfin, il est intéressant de constater qu'un des arguments les plus forts en faveur de la thèse de la spiritualité populaire se trouve dans le développement de mouvements d'opposition à la spiritualité populaire. En effet, la dynamique de cette dernière est vivement contrée par certaines organisations religieuses qui tentent de maintenir des frontières strictes entre la religion et la non-religion, ce que l'on qualifie, dans les études sur la science, de travail sur les frontières (T. Gieryn, 1983). Celui-ci se manifeste notamment par la tentative « fondamentaliste » de maintenir une distance vis-à-vis de la culture " libérale » mainstream. On en trouve un exemple significatif dans les efforts entrepris par le pape Benoît XVI pour distinguer les formes catholiques (et canoniques) des rituels face aux expressions populaires qui se sont abondamment développées sous le pontificat de Jean-Paul II (H. Knoblauch, 2013).

Une fois achevée cette ébauche d'analyse du concept de spiritualité populaire ou de religion communicationnelle, il reste de vastes champs ouverts pour la recherche à venir. Notre esquisse de cadre théorique, en s'appuyant à la fois sur une théorie sociologique de la transcendance et sur le constructivisme communicationnel, montre que l'analyse des formes communicationnelles fournit une base permettant l'investigation empirique de questions centrales concernant les transformations contemporaines du religieux.

De même que l'action communicationnelle implique les sujets, les effets des transformations communicationnelles doivent être spécifiés en fonction du rôle changeant des acteurs. La popularité croissante de la spiritualité indique que 
la subjectivité gagne en pertinence, raison pour laquelle il faut étudier certains aspects de la subjectivité comme les façons dont les acteurs gèrent la pluralité croissante de la religion (A.-S. Lamine, 2004), la mise en avant croissante des expériences de la transcendance (y compris les expériences paranormales ou celles liées à l'usage culturel ou esthétique des narcotiques), des émotions et de l'expression de celles-ci (D. Hervieu-Léger, F. Champion, 1990). À cet égard, il faut reconsidérer le rôle de l'ethnicité, du genre et de la relation aux institutions religieuses (L. Woodhead, 2007). Partant des changements dans la communication, ses matériaux, ses objets et ses espaces (D. Houtman, B. Meyer, 2012), la question se pose de savoir si les concepts d'appartenance aux organisations religieuses, d'identité religieuse et de communauté religieuse en général sont aussi en cours de transformation.

Cette interrogation est particulièrement pertinente dans une période de déstructuration globale de la communication religieuse, accompagnée de recombinaison avec d'autres formes institutionnelles de communication ainsi qu'avec des formes qui excèdent les champs institutionnels et qui deviennent communes à certaines catégories de personnes à l'échelle mondiale - avec les effets que cela entraîne en termes d'inégalités économiques, d'asymétries du pouvoir ainsi que sur les différentes traditions des religions historiques.

Il faut en effet se demander comment et sous quelles formes religieuses la communication transgresse les frontières des institutions religieuses. La transgression de la communication religieuse et l'exigence croissante d'un travail de démarcation au sein des organisations religieuses pourraient contribuer à une dissolution du champ religieux vers une spiritualité populaire, éventuellement liée à des fragments toujours plus étroits de ces organisations; elles pourraient conduire à la (re)formation d'une "religion publique » soutenue par le pouvoir des institutions politiques, économiques, légales ou militaires ; elles pourraient enfin contribuer à la reconfiguration de la communication religieuse par rapport à d'autres formes de communication, institutionnelles ou non, ce qui pourrait conduire à l'abandon de la distinction entre le privé et le public.

Un argument majeur réside dans le fait que ce n'est pas a priori mais seulement par une étude empirique systématique des formes sociales de la religion qu'il est possible de répondre à ces questions cruciales. Étant donné l'abondance des études sur des aspects variés de la communication (tels que les symboles, les rituels, le langage, etc.), le constructivisme communicationnel offre une approche qui permet d'étudier non seulement ce que l'on peut considérer comme la communication religieuse stricto sensu, mais aussi de mettre à l'épreuve la thèse selon laquelle la religion, plutôt que d'être la proie de la modernité, se diffuse dans la société contemporaine sous forme de "spiritualité populaire".

Hubert KNOBLAUCH

Université technique de Berlin hubert.knoblauch@tu-berlin.de 


\section{Bibliographie}

AsAd Talal, 2003, Formations of the Secular. Christianity, Islam, Modernity, Stanford, Stanford Univ. Press.

BARker Eileen, 2004, "The Church Without and the God Within: Religiosity and/or Spirituality ", in D. Marinovic Jerolimov, S. Zrinscak and I. Borowik (eds), Religion and Patterns of Social Transformation, Zagreb, IDZ, p. 23-47.

BaUman Richard, 1983, Let Your Words Be Few. Symbolism of Speaking and Silence among Seventeenth Century Quakers, Cambridge, Cambridge Univ. Press.

Berger Peter L., 1999, "The Desecularisation of the World ", in Berger P.-L. (ed.), The Desecularisation of the World: Resurgent Religion and World Politics, Washington, Eerdmans, p. 1-18.

-, 1974, "Some Second Thoughts on Substantive Versus Functional Definitions of Religion ", The Scientific Study of Religion, 13 (1974), p. 125-133.

Berger Peter L., Luckmann Thomas, 1966, The Social Construction of Reality, Garden City, New York, Doubleday, Inc.

BLOCH Marc, 1924, Les rois thaumaturges : étude sur le caractère surnaturel attribué à la puissance royale particulièrement en France et en Angleterre, Paris, Istra.

Bourdieu Pierre, 1985, "Conclusion : Le champ religieux dans le champ de manipulation symbolique ", in Centre de sociologie du Protestantisme, Strasbourg, (éd.), Les nouveaux clercs. Prêtres, pasteurs et spécialistes des relations humaines et de la santé, Genève, Labor et Fides, p. 255-261.

Bruce Steve, 2002, God Is Dead. Secularization in the West, Oxford, Blackwell.

BüHLER Karl, 1934, Sprachtheorie. Die Darstellungsfunktion der Sprache, Jena, Verlag von Gustav Fischer.

Burke Peter, 1988, Popular Culture in Early Modern Europe. Reprint edition, Hants, England, Wildwood House.

Casanova José, 1994, Public Religions in the Modern World, Chicago, Chicago Univ. Press.

Castells Manuel, 2009, Communication Power, Oxford, Oxford Univ. Press.

Cooley Charles Horton, 1902, Human Nature and the Social Order, New York, Scribner's.

Csordas Thomas J., 2004, "Asymptote of the Ineffable. Embodiment, Alterity, and the Theory of Religion ", Current Anthropology, 45-2, p. 163-185.

Davie Grace, 2002, Europe, the Exceptional Case: Parameters of Faith in the Modern World, Londres, Darton, Longman \& Todd.

DuBy Georges, 1978, Les trois ordres ou imaginaire du féodalisme, Paris, Gallimard.

EIsenstadt Shmuel N., JASPERS Karl, 1999, Fundamentalism, Sectarianism, and Revolution, Cambridge, CUP.

FISKE John, 1989, Understanding Popular Culture, London, New York, Routledge.

Forschungskonsortium WJT, 2007, Megaparty Glaubensfest. Weltjugendtag: Erlebnis, Medien, Organisation, Wiesbaden, VS Verlag.

Gebhardt Winfried, Engelbrecht Martin, Bochinger Christoph, 2005, "Die Selbstermächtigung des religiösen Subjekts ", Zeitschrift für Religionswissenschaft, 2, p. 133152.

GIERYN Thomas F., 1983, "Boundary Work and the Demarcation of Science from NonScience: Strains and Interests in Professional Ideologies of Scientists ", American Sociological Review, 48, p. 781-795. 
Habermas Jurgen, 1981, Theorie des kommunikativen Handelns, Frankfurt am Main, Suhrkamp.

Hanks William F., 1996, Language and Communicative Practices, Boulder, Co, Westview Press.

Hepp Andreas, 2012, Cultures of Mediatization, Cambridge, Polity Press.

Herbrik Regine, KNoblauch Hubert, 2013, «Religion, Emotion, and Mediatization ", in Scheve C., Salmela M. (eds.), Collective Emotions, Oxford, Oxford Univ. Press.

Hervieu-LéGer Danièle, Champion Françoise, 1990, De l'émotion en religion. Renouveau et traditions, Paris, Bayard.

Hervieu-Léger Danièle, 1993, La religion pour mémoire, Paris, Cerf.

HitZler Ronald, 2011, Eventisierung. Drei Fallstudien zum marketingstrategischen Massenspaß, Wiesbaden, VS Verlag.

Honko Laurie, 1968, "Genre Analysis in Folklore and Comparative Religion ", Temenos, 3, p. 48-66.

Houtman Dick, Meyer Birgit (eds.), 2012, Things. Religion and the Question of Materiality, New York, Fordham University.

Huber Stefan, 2009, "Structuring Principles, Operational Constructs, Interpretative Strategies », Religionsmonitor 2008, Gütersloh, Verlag Bertelman Stiftung, p. 17-51.

Husserl Edmund, 1972 [1939], Erfahrung und Urteil, Hamburg, Meiner.

Keller Reiner, Knoblauch Hubert, Reichertz Jo (eds.), 2012, Kommunikativer Konstruktivismus. Theoretische und empirische Arbeiten zu einem neuen wissenssoziologischen Ansatz, Wiesbaden, VS Verlag.

KNoblauch Hubert, 2013, "Benedict in Berlin. The Mediatization of Religion ", in Hepp A., Krotz F. (eds.), Mediatized Worlds: Culture and Society in a Media Age, London, Palgrave, p. 143-158.

-, 2012, "La spiritualité populaire ", in Eckert G., Michon B., Vivarelli C. (eds.), La croyance : de la théorie an terrain, Paris, Hermann, p. 181-206.

-, 2010, "Popular Spirituality ", Anthropological Journal of European Cultures, 19-1, p. 24-39.

-, 2009, Populäre Religion. Auf dem Weg in eine spirituelle Gesellschaft, Frankfurt, New York, Campus.

-, 2008, "Spirituality and Popular Religion in Europe ", Social Compass 55, 2, p. 141154.

-, 2001, «Les expériences du seuil de la mort en Allemagne : la fin d'un déni ? ", Recherches Sociologiques XXXII, no 2, 49-64.

-, 2000, "Populäre Religion. Markt, Medien und die Popularisierung der Religion ", Zeitschrift für Religionswissenschaft, 8, p. 143-161.

-, 1998, «Transzendenzerfahrung und symbolische Kommunikation. Die phänomenologisch orientierte Soziologie und die kommunikative Konstruktion der Religion », in Tyrell H., Krech V., Knoblauch H. (eds.), Religion als Kommunikation, Würzburg, Ergon, p. 147-186.

Lamine Anne-Sophie, 2004, La cohabitation des Dieux. Pluralité religieuse et laïcité, Paris, Presses universitaires de France.

Leroi-Gourhan André, 1964-65, Le geste et la parole, 2 vol., Paris, Albin Michel. Levinas Emmanuel, 1979, Le temps et l'autre, Paris, Presses universitaires de France. 
Luckmann Thomas, 2003, "Moralizing Sermons, Then and Now ", in Fenn R.(ed.), The Blackwell Companion to the Sociology of Religion, New York, Blackwell, p. 120-132. -, 1991, Die unsichtbare Religion, Frankfurt/Main, Suhrkamp.

-, 1990, "Shrinking Transcendence, Expanding Religion?", Sociological Analysis. A Journal in the Sociology of Religion, 50-2, p. 127-138.

-, 1973, «Philosophy, Science and Everyday Life ", in Natanson M. (ed.), Phenomenology and the Social Sciences, vol. 1, Evanston/III, Northwestern Univ. Press, p. 143-185.

-, 1970, " On the Boundaries of the Social World ", in Natanson M. (ed.), Phenomenology and Social Reality, The Hague, Nijhoff, p. 73-100.

-, 1967, The Invisible Religion, New York, The Free Press.

Luhmann Niklas, 2000, Die Religion der Gesellschaft, Frankfurt, Suhrkamp.

LuKES Steven, 1973, Emile Durkheim, Harmondsworth, Penguin.

MEAD Georg Herbert, 1934, Mind, Self, and Society, Chicago, Univ. of Chicago Press.

Muchembled Robert, 1978, Culture populaire et culture des élites dans la France moderne $\left(\mathrm{XV}^{e}-\mathrm{XVIII^{e }}\right)$, Essai, Paris, Flammarion.

Plessner Helmuth, 1975, Die Stufen des Organischen und der Mensch, Berlin, De Gruyter. Pollack Detlef (ed.), 2003, «Deinstitutionalisierung des Religiösen und religiöse Individualisierung in Deutschland », in Säkularisierung - ein moderner Mythos?, Tübingen, Mohr Siebeck.

SAMARIN William J., "The Language of Religion ", in Ammon U., Dittmar N., Mattheier K. J. (eds.), Sociolinguistics, Soziolinguistik, 1. Halbband, Berlin, New York, De Gruyter.

SCHÜTZ Alfred, 2003 [1932], Der sinnhafte Aufbau der sozialen Welt. Eine Einleitung in die verstehende Soziologie, ASW II, edited by Endreß M., Renn J., Konstanz, Wien, Springer.

-, 1966, "The Problem of Transcendental Intersubjectivity in Husserl ", in Collected Papers III, The Hague, Nijhoff, p. 51-83.

-, 1962, "Symbol, Reality and Society ", in Collected Papers I, The Hague, Nijhoff, p. 287-339.

-, 1962a, "Common Sense and Scientific Interpretation of Human Action ", in Collected Papers I, The Hague, Nijhoff, p. 3-47.

SchüTZ Alfred, Luckmann Thomas, 1989, The Structures of the Life World II, Evanston, Northwestern Univ. Press.

Tomasello Michael, 2008, Origins of Human Communication, Cambridge, Massachusetts London, MIT Press.

Troeltsch Ernst, 1992 [1912], The Social Teachings of the Christian Churches, Westminster, Knox.

Weber Max, 1988 [1920], Gesammelte Aufsätze zur Religionssoziologie, Tübingen, Mohr.

Wilke Annette, Moebus Oliver, 2011, Sound and Communication. An Aesthetic Cultural History of Sanskrit Hinduism, Berlin, New York, De Gruyter.

WoodHeAd Linda, 2007, "Why So Many Women in Holistic Spirituality? A Puzzle Revisited », in Flanagan K., Jupp P. C. (eds.), A Sociology of Spirituality, Aldershot, Ashgate, p. 115-125. 


\section{La religion communicationnelle}

Au lieu d'une sécularisation en cours ou d'une re-sacralisation, nous avons assisté, au cours de la dernière décennie, à une transformation rapide de la religion. Afin de bien saisir les nouvelles formes de religion, nous avons besoin d'une théorie qui englobe à la fois la religion publique nouvellement institutionnalisée ainsi que ses formes "visibles" étonnamment privatisées. Cet article propose une esquisse d'une théorie sociologique des religions qui s'appuie sur la notion de transcendance dans la sociologie phénoménologique de Schutz, Berger et Luckmann et la convertit en une notion sociologique de la transcendance comme spiritualité. Après avoir souligné l'ambigü̈té de la notion de transcendance qui se trouve à la racine de la théorie de la religion de Luckmann, l'article délimite le cadre théorique qui permet de saisir la transcendance, c.-à-d. la spiritualité, comme un phénomène social, c.-à-d. le constructivisme communicationnel. Sur cette base, la spiritualité populaire est décrite comme une forme empirique de religion émergeant au cours des dernières décennies, en particulier par rapport à la religion en Allemagne. La dernière partie indique certains des domaines les plus importants de la recherche à venir sur la spiritualité populaire.

Mots clés : Schutz, Luckmann, religion communicationnelle, spiritualité populaire, transcendance.

\section{Communicative Religion}

Instead of an ongoing secularization or a re-sacralization, in the last decade, we are witnessing a rapid transformation of religion. In order to grasp the emerging forms of religion, we are in need of a theory which encompasses both the newly public institutionalized religion as well as its surpringly "visible" privatized forms. This paper offers a sktech of a sociological theory of religion which builds on the notion of transcendence in phenomenological sociology of Schutz, Berger and Luckmann and converts it into a sociological notion of transcendence as spirituality. After having highlighted the ambiguity of the notion of transcendence which lies at the root of Luckmann's theory of religion, the article delineates the theoretical frame which allows to grasp of transcendence, i.e. spirituality, as a social phenomenon, i.e. communicative constructivism. On this basis, popular spirituality is outlined as an empirical form of religion emerging in the recent decades, particularly with respect to religion in Germany. The final part indicates some of the most important areas of future research on popular spirituality.

Key words: Schutz, Luckmann, communicative religion, popular religion, transcendence.

\section{La religión comunicacional}

En lugar de una secularización o una re-sacralización en curso, en la última década, estamos asistiendo a una rápida transformación de la religión. Con el objetivo de comprender las formas emergentes de la religión, necesitamos una teoría que abarque tanto la nueva religión pública institucionalizada, como sus sorprendentes formas "visibles" privatizadas. Este artículo propone el esbozo de una teoría sociológica de la religión que se construye sobre la noción de trascendencia en la sociología fenomenológica de Schutz, Berger y Luckmann, y la convierte en una noción sociológica de trascendencia como espiritualidad. Luego de destacar la ambigüedad de la 
IO4 - ARChives De SCIENCES SOCIALES DES RELIGIONS

noción de trascendencia que subyace a la teoría de la religión de Luckmann, el artículo delinea el marco teórico que permite dar cuenta de la trascendencia, es decir la espiritualidad, como un fenómeno social, es decir comunicativo constructivista. Sobre estas bases, la espiritualidad popular es destacada como una forma empírica de religión emergente en las décadas recientes, particularmente con respecto a la religión en Alemania. La parte final indica alguna de las más importantes áreas de investigación futura en espiritualidad popular.

Palabras clave: Schutz, Luckmann, religión comunicacional, espiritualidad popular, trascendencia. 